International Journal of Engineering \& Technology, $7(2.7)(2018) 183-186$
International Journal of Engineering \& Technology
WPC
Website: www.sciencepubco.com/index.php/IJET
Research Paper

\title{
Facial recognition enabled smart door unlock system
}

\author{
M Vamsi Krishna*, A Bhargav Reddy, V Sandeep \\ Department of Electronics and Computer Science Engineering, KLEF, Vaddeswaram \\ *Corresponding author E-mail: vamsi.mysore@gmail.com
}

\begin{abstract}
To verify that our daily life is going in a secure way. Lot of research programmers are going on in this entire society. The turning point comes through the internet of things, industry has been emerged with the lots of elements provided from IOT. We can able to connect our daily life things or objects with this had successfully evolved lots of things. This Facial recognition door unlock system is a process is which will detect the face and identifies the among people. People are having different types of face cut, in that particularly there are many unique faces which are different from each other which inspired us, from that concept this process has been established. Our main aim to create the smart door system to a house, that will secure the house and all your personal things at your home. In this concept of our system we have been used alive web camera in the front side of the door, along with the display monitor. this web camera shows the owner/particular viewer the whom the house is his control, this shows the person who stood front of the door, the system is setup the voice output is being processed by the processor that which is used to show the answers/instructions as the output on the screen. We are using a stepper motor that which is used to lock/open then the by sliding method, so that a normal person stand in front of the door and access it. This process is done through this Microsoft face API application. The display is being operated on a Microsoft Visual Studio application.
\end{abstract}

Keywords: Facial Recognition, Microsoft Face API, Door Unlock, Stepper Motor, Live Streaming.

\section{Introduction:}

Now-A-days in this world is connected to the smart devices there is a crucial need that is to improve the existing objects and make them smart. Especially to our door locks. To make any object smart enabled we need to explore all its existing drawbacks and add some extra specifications. The major disadvantages in a normal door locking system is that anybody can open a normal door by a duplicate key and it's very difficult if we want our friends and family to get into our house. This is why we cannot decrease these problems. So just change this normal door locking system into a facial recognition enabled smart door lock, which we can open the door whenever we want, So this implementation has come where devices can interact with the users and at the same time verify the safety and keeping them smart. The main concept is that to design and model this Facial recognition door unlock in advanced knowledge of the microcontrollers and interfacing the requirements, as the Raspberry Pi 3 computing is used and been interfaced with the different devices along with the development of application to develop a desktop application. By a thorough study on the libraries and there functions we has generate the code, we does a most dependable and perfect facial recognition with the new and effective use of the hardware. It is how I initiated and making life easy and interconnected with objects. This will contribute a major change in Home Security.

\section{Existing Model}

Since 2009 there has been a drastic change in the Machine Learning, IOT, Artificial Intelligence, Big Data analytics which are all with the common to make works easier, self-learning and inter- connected with all smart devices. An intensive literature over the smart locking systems has been done and door locks. The fault in existing model is unnecessary relying and lack of security. Our model helps in removing such kind of faults. Present systems are lagging with lack of functionalities for that sake, We have provided many functionalities which make more easy to understand and very flexible to use. The main objective of proposed system is to make a working model smart door unlocking with secured and informative about the entry of people and providing a live streaming. This system will help in key research area of Neural Networks and IOT APIs and protocols.

\section{Proposed Methodology}

In the present system we have been using the equipment's of

Raspberry Pi 3

Web Cam

GSM module

Stepper motor

Monitor Display

Door Bell

Breadboard, Push Buttons and Connecting Wires

\section{Methodology and Working of present Sys- tem}

To start this project at first we need a Raspberry Pi 3, the setup of Raspberry pi3 should be done with Windows 10 IOT. Then we interface a display to the ARM processor. The camera is connected and interfaced with the processor through the input port, which captures the image of the person who is entering the home, the 
GSM module provided to the Pi will help in intimating the owner of the house about the status that who has come. The IR sensor provided to make count of the persons how many has entered the home this helps in owner to know the information that who has entered the home. We provider live streaming process through the app and also helps the owner to permanently lock door until he link data to the cloud through app, the Raspberry Pi will always scan the video footage and stored in cloud which helps the owner to view the live status at his home. If any intruder tries to enter the home it will send a message to the owner of the house and rings the bell.

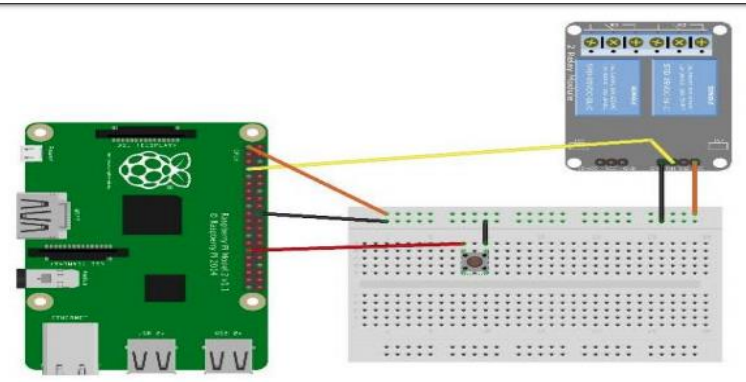

Fig. 1: Circuit Diagram

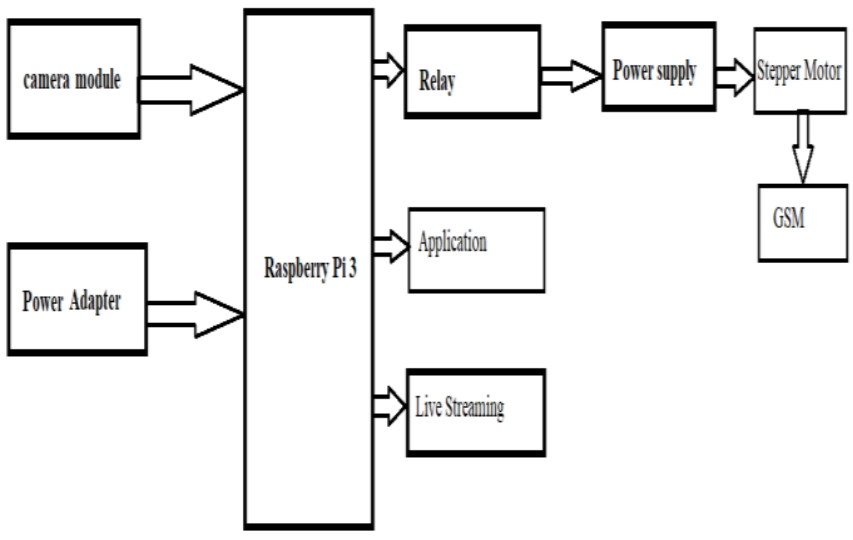

Fig 2: Block Diagram

\section{Raspberry pi 3}

It is a kind of development board which has the modules like WIFI and Bluetooth comes on board, we can connect any type of sensors to Raspberry Pi3 through the GPIO pins general the Raspberry $\mathrm{Pi}$ is coded with Python based on the sensors used and can call the data to the cloud it is one of the famous development board in the market. it has 40 GPIO pins 4 USB ports , 1 LAN port , 1 port for power supply and It has on board provided with $1 \mathrm{~GB}$ RAM with a quad core cortex A53 CPU running on an $1100 \mathrm{Meg}-$ ahertz it supports and external memory where the operating system of the Raspberry Pi exist according to the Benchmarks there were Raspberry Pi 3 is $80 \%$ faster than all the previous development boards in the Pi series.

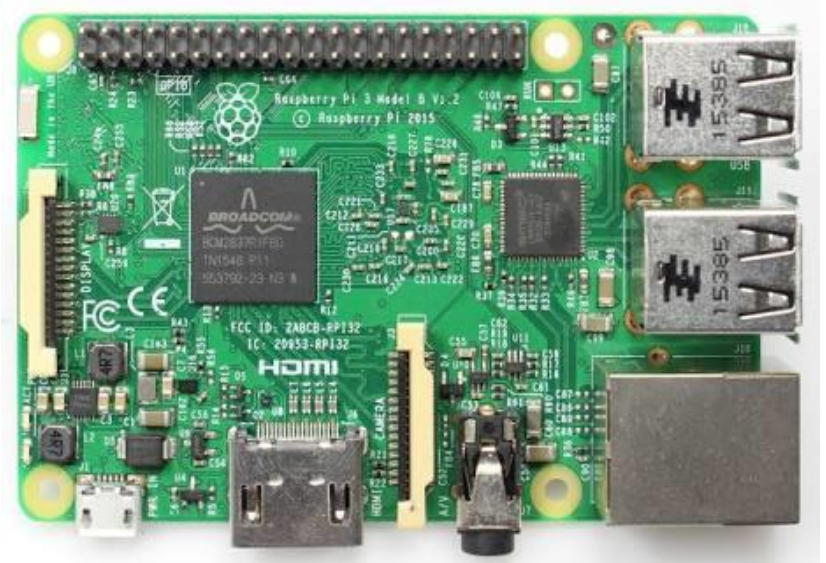

Fig. 3 Raspberry Pi 3

\section{Face Recognition:}

For face recognition in this project we used Microsoft face recognition API where it can detect the user from unique identities like colour, height, age, gender, smileff, head pose, facial hair, moustache, glasses, anger, etc.,.

this face recognition API can be used through C\# on Microsoft Visual Studio where it can scan data and store in Microsoft Azure database.

the face data can be stored in database in the form of binaries when the user face is detected which captures the face in three dimensional wave like Pitch, Roll, Yaw and complaints it with the data which is stored in the database as every user I have an unique face identity so it is impossible to break the lock.

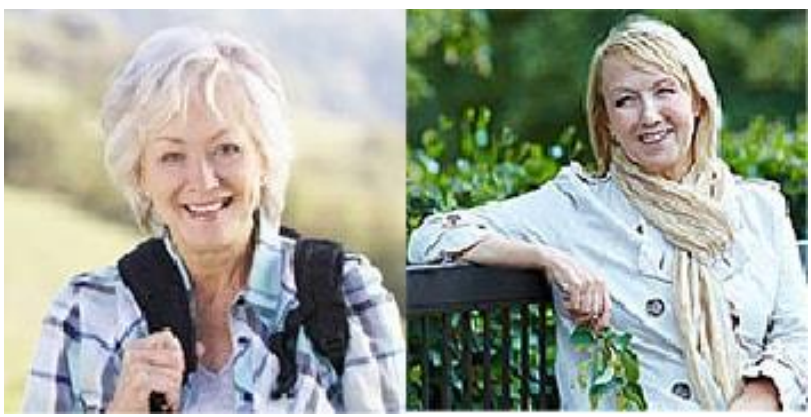

Fig. 4 Face Recognition API

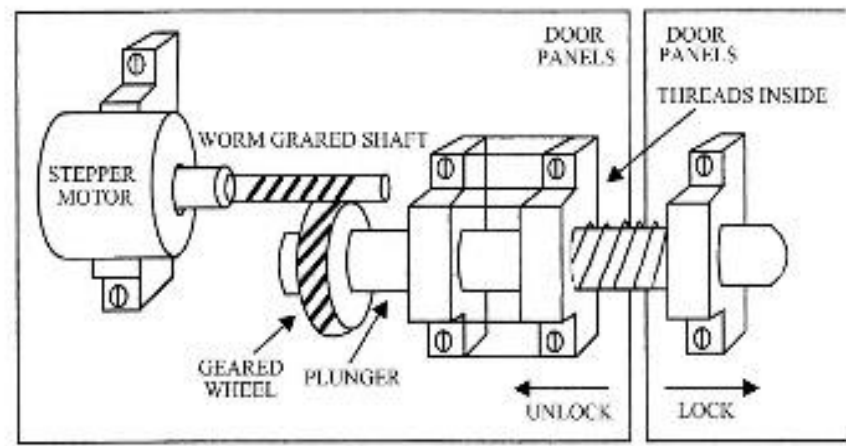

Fig. 5: Stepper Motor Mechanism

\section{Unlocking Door}

For unlocking the door use stepper motor where when the Face data is read and the door is unlocked for unlocking we should need a stepper motor interface with the Pi where stepper motor starts rotating for a specified amount of time based on the Delay provided until the door opens, after a certain amount of time the door gets automatically locked.

\section{IR sensor}

Infrared sensor is an electronic instrument which helps in Sensing the things like MTN detecting the radiation. it is also capable of measuring heat or speed and it is also used to send data from one node to another node.

here in the project used IR sensor for transmitting data from one node to another node when obstacle/ user hits the radiation it 
makes a count. By this count it helps the owner of the house to know how many persons are entering now.

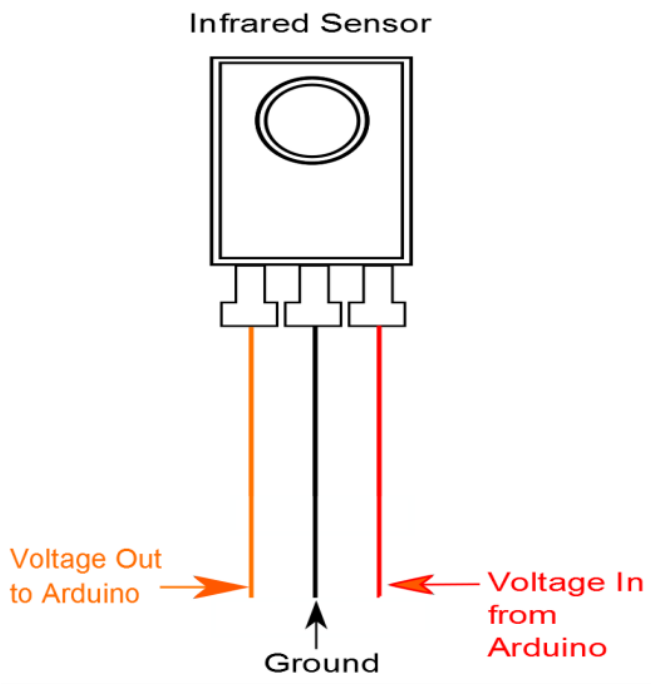

Fig. 6: IR sensor

\section{GSM module:}

GSM module is like a SIM tray for mobile the GSM module helps in connecting the SIM card to the Raspberry Pi using that we can stay connected to the network this helps in sending an important message required to be informed to the owner, the message are previously fixed in the software and detecting any Intruder or any person more than one and then the home the GSM module will intimate the owner so that he can be cautious about the information and verify the person entering unknown person or an unknown.

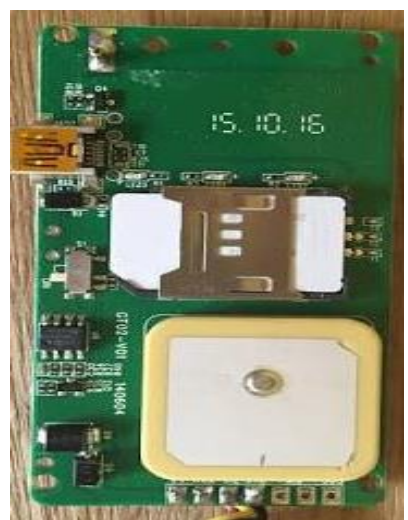

Fig. 7: GPS Moodule

We can explain the whole project on the basis of five major divisions:

1. The person who needs to enter first press the doorbell then the Camera module provided will captures the image of the person and find out the face of the person and crops the image which will be sent to Microsoft face recognition API which was connected to the Microsoft Azure cloud setup. the fish is identified from the database which was stored previously in the cloud. if the face matches with any user in the database then plays audio of "welcome (Name Of the person)" the processor which is controlling the relay module will open the door.

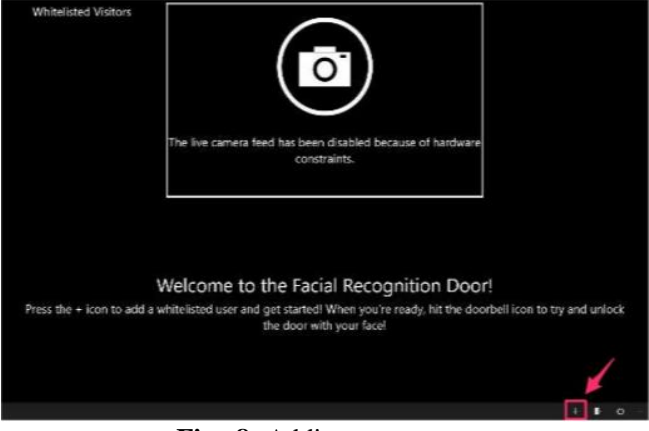

Fig. 8: Adding users

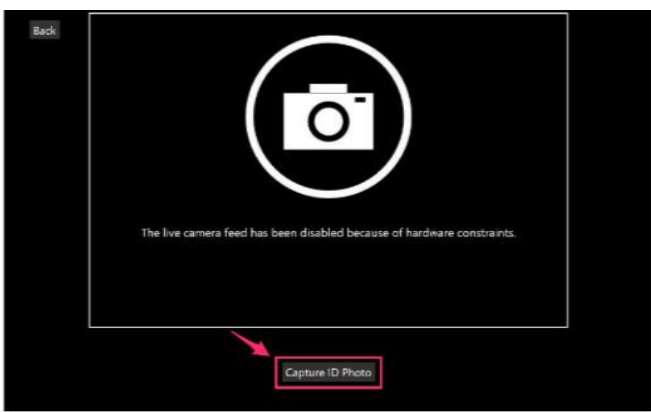

Fig. 9: Adding users Functionality

2. The second major part of the project is adding users to the database. To add new user, we should press the add user button the camera module which was attached to the board will turn on and captures the image of the person who is in front of the camera after capturing the image it will ask for the name of the person and that's in to the database on cloud which helps in recognising the face.

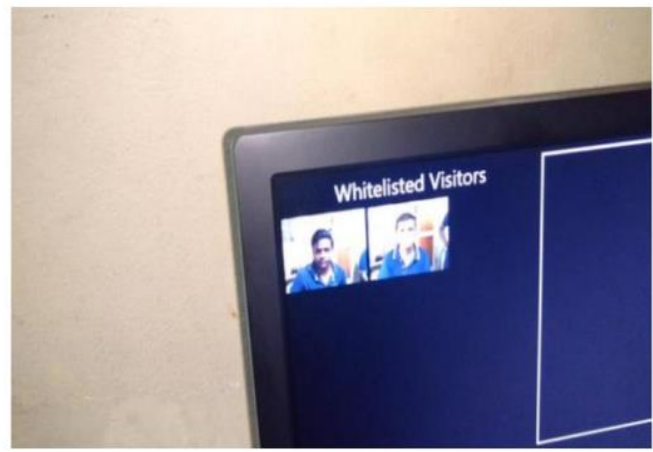

Fig. 10: White list User

3. The third main thing we need to do is we should count the number of the person entering the home for this we need to take an IR sensor. if the IR sensor is On and if any obstacle hit the rays emitted by the sensor then it makes a count so that we can know how many persons are entering inside.

4. The fourth main thing is who should message the owner of the home through the GSM module provided that someone has entered the home if the person is an Intruder then then it should stop him from entering the home and if the person is already registered in the database then it should send the name of the person who is entering the home.

5. The fifth main thing we should do in this project is we should provide a live streaming video to the owner through the app provided for the streaming of live video this helps to verify the status of any Intruder entry into the home or not. This also helps owner to in locking door permanently through the app by disconnecting face data temporarily and then connect the data when want to. 
We started with a Raspberry Pi3 which is being booted with windows 10 IOT version, the display is being interfaced with the ARM by using assembly language to interface display, and camera module is also interfaced with the ARM processor to provide the input of person stood before the door and to capture the image to apply facial recognition module by Raspberry Pi. Then the door will be automated by giving an instruction then the door will be opened. we had decided to add a special module of the IR sensor to detect the number of persons who had been entered in to the room/house. Then it will help in the malfunction of the stepper motor.

\section{Conclusion}

In this work, automatic door unlocking system we use face recognition API, which is done by Neural Networks. After a face recognition is successful the Raspberry pi3 controller. The user can have a freedom to lock door from application. The user can have a freedom if the door should open or keep it locked. This system will also provide user to live stream the video. We utilize Local Binary Patterns Histogram algorithm for the face recognition prepare. Additionally, Alarm will ring for unauthorized access. The created plan is shoddy, quick, and profoundly dependable and gives enough adaptability to suit the prerequisites of various frameworks.

\section{References:}

[1] 1.Jie-Ci Yang et. all An Intelligent Automated Door Control System Based on a Smart Camera

[2] 2.S. Nazeem Basha et all An Intelligent Door System using Raspberry pi

[3] 3.Chaitanya Rane Password Based Door Locking System Using GSM

[4] 4. Pratiksha Misal et.all Door Locking/Unlocking using SMS Technology with GSM/GPRS technology

[5] 5.Anoop Mishra "Embedded Image Capturing \& Digital Converting Process using Raspberry pi

[6] 6.Ole Helvig Jensen, Kongens Lyngby, "Implementing the Viola-Jones Face Detection Algorithm" in IMM. DTU, IMM-M.Sc. ISBN 87-643-0008-0,ISSN 1601-233X. 